\title{
Efecto del Ultrasonido y Campos Magnéticos en la Carne de Lomo Atún (Thunnus albacares)
}

\author{
Lorenzo Fuentes $^{(1) *}$, Diofanor Acevedo ${ }^{(1)}$ y Víctor M. Gélvez ${ }^{(2)}$ \\ (1) Universidad de Cartagena, Facultad de Ingeniería, Programa de Ingeniería de Alimentos, Avenida el \\ Consulado, Calle 30 No. 48-152. Cartagena, Bolívar-Colombia (e-mail: Ifuentesb@unicartagena.edu.co) \\ (2) Universidad de Pamplona, Facultad de Ingeniería, Programa Ingeniería de Alimentos, Km 1 Via \\ Bucaramanga. Norte de Santander, Colombia.
}

*autor a quien debe ser dirigida la correspondencia

Recibido Abr. 13, 2015; Aceptado Jun. 23, 2015; Versión final Ago. 1, 2015, Publicado Abr. 2016

\begin{abstract}
Resumen
El objetivo de este estudio fue evaluar el efecto del ultrasonido de alta intensidad y los campos magnéticos sobre el $\mathrm{pH}$, el perfil de textura y el color en la carne de lomo de atún. Se aplicó ultrasonido de alta intensidad $\left(37 \mathrm{kHz}, 25 \mathrm{~W} / \mathrm{cm}^{2}\right)$ con un equipo de ultrasonido Elmasonic $\mathrm{E}$ (modelo $\mathrm{E} 60 \mathrm{H}$ ) y campos magnéticos con una intensidad de 1 Tesla, utilizando un equipo generador de campo magnético Scoli, $8 \mathrm{~A}$ $\mathrm{N} 240$ ( $R=2,8 \Omega$ Ohmio). Se realizó un diseño al azar con un solo factor (tiempo de exposición a ultrasonido y campos magnéticos) en 4 niveles $(0,3,4$, y $5 \mathrm{~min})$. Los resultados mostraron que el tratamiento en los parámetros de dureza y elasticidad con ultrasonido presentaron diferencias significativas comparados con la muestra control $(p<0,05)$. En síntesis, la aplicación de ultrasonidos y campos magnéticos afectó el perfil de textura y las coordenadas colorimétricas en la carne de atún.
\end{abstract}

Palabras clave: alta intensidad, medición de color, perfil de textura, $\mathrm{pH}$

\section{Effect of Magnetic Fields on Ultrasound and Loin Tuna (Thunnus albacares)}

\begin{abstract}
The aim of this study was to evaluate the effect of high intensity ultrasound and magnetic fields on the $\mathrm{pH}$, the texture profile and color in tuna meat. High intensity ultrasound $\left(37 \mathrm{kHz}, 25 \mathrm{~W} / \mathrm{cm}^{2}\right)$ were applied with a heated ultrasonic device Elmasonic $\mathrm{E}(\operatorname{model} \mathrm{E} 60 \mathrm{H})$ and magnetic fields with an intensity of 1 Tesla were produced using magnetic field generator Scoli, 8A N240 $(R=2.8 \mathrm{Ohm} \Omega)$. A randomized design was performed using a single factor (time of exposure to ultrasound and magnetic fields) in 4 levels $(0,3,4$, and $5 \mathrm{~min}$ ). The results showed that the ultrasound treatment parameters in hardness and elasticity showed significant differences with the control sample $(p<0.05)$. In brief, application of ultrasound and magnetic fields affect the texture profile and the colorimetric coordinates in tuna meat.
\end{abstract}

Keywords: high intensity, color measurement, profile of texture, $\mathrm{pH}$ 
Efecto del Ultrasonido y Campos Magnéticos en la Carne de Lomo Atún (Thunnus albacares) Fuentes

\section{INTRODUCCIÓN}

La conservación de los alimentos ha sido el objetivo primordial de la industria procesadora de alimentos, puesto que garantiza la seguridad y la calidad de estos (Pereira y Vicente, 2010). La aplicación del calor a través de las operaciones de tratamientos térmicos, que entre otros incluye la pasterización, esterilización, secado y evaporación ha sido la práctica común de las industrias con el fin de garantizar la seguridad microbiológica de sus productos (Pereira y Vicente, 2010). En la última década las tecnologías no térmicas que se utilizan para inactivar microorganismos se han desarrollado en respuesta al interés de ser utilizado en productos frescos y naturales. Este tipo de tecnologías Las nuevas tecnologías no térmicas, como ultrasonido (US), alta presión hidrostática (HHP), Campos eléctricos pulsados (PEF) y el calentamiento óhmico $(\mathrm{OH})$ tienen la capacidad de inactivar microorganismos a temperaturas cercanas a la del ambiente evitando la degradación de los componentes en los alimentos, y por lo tanto la preservación de la calidad sensorial y nutricional de los productos alimenticios (Pereira y Vicente, 2010).

Por lo tanto estas tecnologías de conservación de alimentos e innovadores pueden proporcionar nuevas oportunidades para el desarrollo de estrategias integradas, dirigido a los consumidores para el desarrollo de alimentos sanos, seguros y de alta calidad (Turantaş et al., 2015). Uno de estos métodos que se utilizan para procesar los alimentos es el US de alta potencia. EI US es una forma de energía generado por una onda de presión de sonido con frecuencia superior $(>20 \mathrm{KHz})$, que es límite superior del rango de audición humana basado en el nivel de frecuencia, el US se divide en dos categorías: la de baja intensidad y la energía de alta potencia (AP). EI US de AP se puede emplear a un gran número de aplicaciones de procesamiento industrial, incluyendo la elaboración de alimentos y la seguridad alimentaria en áreas relacionadas (Bilek y Turantaş, 2013; Arroyo et al., 2011; Barbosa-Cánovas et al., 2011; Patist y Bates, 2010; Rastogi, 2010).

El pescado es bien conocido como una fuente saludable de proteínas dietéticas ricas en aminoácidos esenciales, grasas, vitaminas y ácidos grasos insaturados que están asociados con la reducción de contraer trastornos neuronales (Jump et al., 2012) y enfermedades cardiovasculares, atribuido a la presencia de ácidos grasos poliinsaturados, tales como el ácido eicosapentanoico y el ácido decosahexaenoico (Omega 3) (FAO, 2006). El pescado es una valiosa fuente de macroelementos esenciales como el $\mathrm{Na}, \mathrm{K}, \mathrm{P}$ y Mg, que desempeñan un papel clave en la salud humana (Boufleur et al., 2013). Además el atún constituye un alimento de elevada calidad nutricional, sus proteínas contienen todos los aminoácidos esenciales requeridos por el organismo y presenta un importante contenido en vitaminas y minerales, (FAO, 2006). Uno de los objetivos de los ingenieros y los científicos de alimentos en las últimas dos décadas ha sido buscar tecnologías alternativas de conservación que sean amigables con el medio ambiente, de bajo costo y que conserve los atributos de calidad del producto alimenticio (Barbosa-Cánovas y Bermúdez, 2010). Una variedad de nuevas tecnologías no térmicas, como el US, la irradiación y la aplicación de campos magnéticos (CM) han sido comercializadas y ofrecidas al consumidor muchas de estas ventajas (BarbosaCánovas y Bermúdez, 2010).

Especialmente en los últimos años, algunos investigadores han examinado el potencial de los US, por sí mismo en combinación con otros métodos para aplicaciones que van desde la mejora de los criterios de calidad con la modificación de las propiedades funcionales de las proteínas, la mejora de la vida útil, la reducción del cloruro de sodio y el aumento del rendimiento de cocción para determinar características de la canal y la composición de la carne. Por lo tanto se puede destacar que el US ha sido objeto de interés para la industria de la carne, sin embargo hay muy poca información disponible sobre los efectos del US en las características de la calidad de carnes y de los agentes patógenos (Condón et al., 2011; Haughton et al., 2012). El tratamiento con CM y electromagnéticos es una de las alternativas que se propone para el tratamiento de alimentos, especialmente en la eliminación de los microorganismos patógenos que se presentan en los mismos (Mesa et al., 2012).

De la mayoría de los métodos de conservación no térmicas, sólo el campo magnético puede aplicarse en la estimulación de microorganismos de interés y en la variación de varias propiedades físico-químicas como la densidad y viscosidad (FDA, 2000). El campo magnético (CM) es obtenido a partir de corriente eléctrica que pasa a través de una bobina, campo electromagnético (CEM). Según la frecuencia de las oscilaciones de la corriente por segundo $\left(\mathrm{Hz}=\mathrm{s}^{-1}\right)$, se clasifica en campo magnético estático (CME) y oscilante $(\mathrm{CMO})$ que a su vez se califica como CMO de baja o alta frecuencia (FDA, 2000). Los CM estáticos y oscilantes han sido explorados por su potencial como métodos de inactivación microbiana. Para CEM, la intensidad del campo magnético es constante con el tiempo, mientras que un $\mathrm{CMO}$ se aplica en forma de amplitud constante o en descomposición de ondas sinusoidales (Villalpanda et al., 2011). El objetivo de este estudio fue evaluar el efecto del US de alta intensidad y los $\mathrm{CM}$ sobre el $\mathrm{pH}$, el perfil de textura y la medición de color en la carne de lomo de atún (Thunnus albacores) a los $0,3,4$ y 5 minutos. 


\section{MATERIALES Y MÉTODOS}

La metodología se presenta por secciones para mayor claridad, en el siguiente orden: obtención de la materia prima, acondicionamiento de la muestra, aplicación de US de alta intensidad y $\mathrm{CM}$, $\mathrm{pH}$ de la carne de atún, análisis de perfil de textura TPA, medición de color, análisis estadísticos.

Obtención de la materia prima

El atún aleta amarilla Thunnus albacares, es una especie oceánica epipelágica cuya distribución es amplia, cubriendo los mares tropicales y subtropicales, distribuidas en el oceáno pacífico-oriental, desde el Sur de California hasta Perú (Baque-Menoscal et al., 2012). El lomo de atún de la especie Thunnus albacares, fue adquirida por la empresa Atunec S.A., ubicada en la Zona Franca Industrial de Barranquilla-Atlántico, Colombia.

\section{Acondicionamiento de la muestra}

La carne fue identificada en bolsas plásticas y se transportaron refrigeradas a $4{ }^{\circ} \mathrm{C}$ hasta su posterior análisis, después se adecuo retirando las partes indeseables (tendones y piel) y se cortaron en cubos de $2 \mathrm{~cm}^{3}$; posteriormente se empacaron al vacío (90\%) en un equipo Henkovac y se almacenaron en refrigeración a $4^{\circ} \mathrm{C}$.

\section{Aplicación de US de alta intensidad y CM}

Carne de lomo de atún se expuso a US de alta intensidad $\left(37 \mathrm{kHz}, 25 \mathrm{~W} / \mathrm{cm}^{2}\right)$ durante $0,3,4$ y $5 \mathrm{~min}$, con una potencia de calentamiento de 400W, utilizando el equipo (Elmasonic E Modelo: $\mathrm{E} 60 \mathrm{H}$ ) con regulación de tiempo de intensidad y temperatura, empleando como medio de transmisión agua desionizada de alta pureza. Estos ensayos se realizaron por triplicado.

En la aplicación por CM se usó el equipo (Scoli, $8 \mathrm{~A} N 240, \mathrm{R}=2,8$ ' $\Omega$ Ohmio) a una intensidad de 1 Tesla, estos ensayos se realizaron por triplicado y los tiempos de tratamientos fueron de $0,3,4$ y 5 minutos a temperatura de $18^{\circ} \mathrm{C}$.

\section{pH de la carne de atún}

La determinación fue realizada en un pH-metro ( $\mathrm{pH}$-Schott) debidamente calibrado siguiendo la metodología de la A.O.A.C. (1990). La medición se realizó por triplicado para cada una de las muestras de carne de atún control y tratadas.

\section{Análisis de Perfil de Textura TPA}

El análisis instrumental de textura (TPA) de la carne de lomo de atún, se realizó en un texturómetro Texture Analyser (TA-Plus, Lloyd Instruments) utilizando el software Nexigen. Para el ensayo, se empleó una celda circular de $10 \mathrm{~cm}$ de diámetro y se realizaron las siguientes variaciones: Distancia de 20mm, Fuerza Trigger de $7 \mathrm{gf}$; velocidad del test de 4,5mm/s y compresión a $65 \%$ de la altura inicial del producto deformación. Se ejecutaron 10 mediciones de textura para cada una de las muestras control y tratadas.

\section{Medición de color}

Se utilizó la metodología descrita por Campo y Gélvez (2011), empleando el modelo de color CIELAB (con apertura del diámetro $8 \mathrm{~mm}$, plato blanco de referencia, iluminación estándar D65 (representa la luz del día y un observador estándar de $2^{\circ}$ ), se colocó una muestra de HEL homogénea en una cápsula de cuarzo midiendo tres parámetros de color por reflactancia: L (claridad 0 a 100), a* (componentes de rojo-verde, 6060), b* (com-137 ponente amarillo-azul, 60-60). Las lecturas fueron realizadas en un espectrofotómetro de esfera serie sp60 X - Rite (Grand Rapids, Michigan, U.S.A) con el software X-Rite Color® Máter espacio previamente calibrado. Los análisis se realizaron por triplicado con tres repeticiones cada uno

\section{Análisis estadísticos}

Se realizó un diseño completamente al azar con un solo factor (tiempo de exposición a US y CM en 4 niveles $(0,3,4$, y $5 \mathrm{~min})$, con tres repeticiones para cada tratamiento con US y CM, para un total de 24 unidades experimentales (Montgomery, 2004). Se realizó un análisis de varianza (ANOVA) y se aplicó la prueba post hoc de diferencia mínima significativa (DMS) fijando un nivel de significancia a $p<0,05$. Los resultados fueron procesados utilizando el paquete de software estadístico (SPSS versión. 14). 


\section{RESULTADOS Y DISCUSION}

Efecto del tratamiento con US sobre el $\mathrm{pH}$

En la figura 1 se observa que por efecto de la aplicación de US, hubo variación del pH en la carne de lomo de atún (Thunnus albacares), las letras $a, b, c$ y d indican los tiempos de exposición de las muestras. El tratamiento durante 3 minutos no afectó el pH con relación a la muestra control. No obstante, el tratamiento durante 5 minutos afecta significativamente $(p<0,05)$ disminuyendo el $\mathrm{pH}$ y, por lo contrario el tratamiento por 4 minutos aumenta significativamente el $\mathrm{pH}$ en 0,34 unidades respecto al control. A su vez Jayasooriya et al., (2007), estudiaron el efecto de US en 2 especies de bovino indicando que a medida que aumentaba el tiempo de exposición a US el pH también lo hacía, variando desde 5,37 hasta 5,58 y desde 5,40 hasta 5,71 para cada especie respectivamente, para tiempos desde 0 a 250seg. Mecanismos propuestos incluyen daños a la estructura celular liberando iones en el citosol y los cambios en la conformación de proteínas ocultando los grupos ácidos (Jayasooriya et al., 2007).

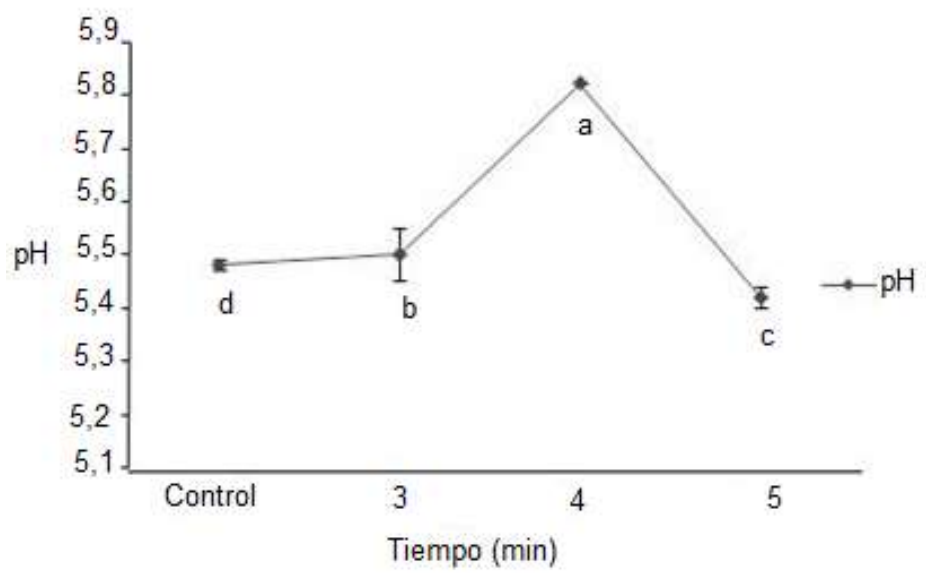

Fig.1: Efecto del US sobre el pH de la carne de lomo de atún

El uso de US también ha sido eficaz en la inactivación de microrganismos, las ondas ultrasónicas de alta amplitud producen cavitación, que es la formación de burbujas o cavidades en el medio. La cavitación estable provoca una corriente de burbujas dentro del campo sonoro que son abrasivas a la superficie de la membrana de las células ocasionando que esta se fracture (San Martín et al., 2001a). Los métodos alternativos de procesamiento de alimentos que tienen mínima influencia en la calidad de los alimentos se han vuelto más importante debido al aumento de la demanda del consumidor para los alimentos mínimamente procesados. La tecnología del US es una alternativa que ha mostrado ser prometedor en este campo. Con la técnica de US, alta presión, cizalladura, y un gradiente de temperatura son generados por US de alta potencia (de 20 a $100 \mathrm{kHz}$ ), que puede destruir las membranas celulares y el ADN, lo que conduce a la muerte celular (Chen et al., 2012). Un concepto relativamente nuevo es el tratamiento antimicrobiano que se ha propuesto, el cual implica el efecto combinado de presión y US (manosonicación), calor y US (termosonicación) o la combinación de calor, presión y US (Manotermosonicación), estos son probablemente los mejores métodos para inactivar los microorganismos (Alarcon-Rojo et al., 2015). Sin embargo algunos efectos, incluyen cambios adversos en la estabilidad del color (Stadnik, 2009), se cree que estos cambios son causados por alteraciones físicas y químicas de las proteínas de la carne (McDonnell et al., 2014), hasta la fecha pocos autores han modelado la cinética de inactivación de esporas microbiana a través de US, los factores críticos que determinan la eficacia de esta tecnología en términos de inactivación microbiana incluyen la amplitud de las ondas ultrasónicas, tiempo de exposición, la temperatura de tratamiento y el tipo de microorganismo (Evelyn y Silva, 2015).

\section{Efecto del tratamiento con US sobre la textura}

En la Tabla 1 se muestran los resultados del análisis de varianza y la comparación de medias a través de la prueba DMS para las variables analizadas. El análisis de perfil de textura se dirige en cuatro variables que se analizan a continuación: dureza, cohesividad, elasticidad y masticabilidad. De la evaluación del perfil de textura en la carne de lomo de atún (Thunnus albacares), se relacionan los resultados en la Tabla 1, la cual muestra que en los parámetros de dureza y elasticidad hubo diferencias significativas entre la muestra control y la carne expuesta a US con valores promedios del $70,45 \%$ y $310,15 \%$ respectivamente, sin embargo durante el tratamiento con US no hubo diferencias significativas entre las muestras. Esto significa que la dureza y la elasticidad fueron estadísticamente iguales entre todos los tratamientos a US. 
Tabla 1: Efecto del US sobre la textura en la carne de lomo de atún. *Letras distintas en una misma columna, indican diferencias estadísticamente significativas $(p<0,05)$ según test DMS.

\begin{tabular}{|c|c|c|c|c|}
\hline \multirow{2}{*}{ Tratamiento } & \multicolumn{4}{|c|}{ Parámetros del análisis de perfil de textura } \\
\cline { 2 - 5 } & Dureza $(\mathrm{N})$ & Cohesividad & Elasticidad & Masticabilidad (Kg) \\
\hline Control & $73,04 \pm 5,1^{\mathrm{a}}$ & $0,18 \pm 0,00^{\mathrm{a}}$ & $1,05 \pm 0,13^{\mathrm{a}}$ & $0,23 \pm 0,00^{\mathrm{a}}$ \\
\hline US 3 min & $54,66 \pm 8,0^{\mathrm{b}}$ & $0,11 \pm 0,03^{\mathrm{a}}$ & $3,55 \pm 0,82^{\mathrm{b}}$ & $0,18 \pm 0,00^{\mathrm{b}}$ \\
\hline US 4 min & $51,94 \pm 6,9^{\mathrm{b}}$ & $0,14 \pm 0,35^{\mathrm{a}}$ & $3,33 \pm 0,71^{\mathrm{b}}$ & $0,14 \pm 0,00^{\mathrm{c}}$ \\
\hline US 5 min & $47,35 \pm 5,3^{\mathrm{b}}$ & $0,11 \pm 0,36^{\mathrm{a}}$ & $2,89 \pm 0,81^{\mathrm{b}}$ & $0,11 \pm 0,00^{\mathrm{d}}$ \\
\hline Valor-p & $0,005<0,05$ & $0,983>0,05$ & $0,0075<0,05$ & 0,0000 \\
\hline DMS, $\alpha=0,05$ & 66,63 & 0,52 & 3,75 & 0,17 \\
\hline
\end{tabular}

También se observa que la cohesividad no mostró un efecto significativo entre la muestra control y el lomo de atún, esto indica que el US no afectó este parámetro de textura. Por otra parte la masticabilidad fue el único parámetro que mostró un efecto significativo entre todas las muestras analizadas, aludiendo que el US tiene un efecto negativo cuando se expone a este tipo de tecnología.

Hernández y Gélvez (2011) reportaron que el efecto de US sobre el perfil de textura (TPA) incrementó en los parámetros de dureza, cohesividad, gomosidad y firmeza en las propiedades del huevo entero líquido, siendo la dureza y la masticabilidad mayor que el control en un $43,39 \%$ y un $65 \%$ respectivamente. Por otra parte McDonnella et al., (2014) mostraron valores medios de TPA para la dureza (N), masticabilidad (Kg), cohesividad, y elasticidad, que fueron 109,$4 ; 230,3 ; 0,9$; y 5,7 respectivamente para una carne de cerdo a nivel de planta piloto. Entre los métodos indirectos de medición de la textura de los alimentos, la tecnología de US proporciona una de las bases de una técnica no destructiva, rápida y fiable para la correlación de los índices y las características específicas relacionadas con la calidad durante el crecimiento y maduración, y en el curso de almacenamiento y vida útil, hasta la preparación para el consumo (Chen y Opara (2013). La investigación en las últimas décadas ha demostrado los beneficios potenciales del uso de tratamiento de US como una técnica para mejorar la ternura y las propiedades sensoriales de la carne debido a que acelera el proceso de maduración, favoreciendo atributos en el sabor y textura de esta, además se reducen los costos del producto terminado (Stadnik et al., 2011).

La calidad de la carne depende principalmente del aroma, sabor, apariencia, textura y jugosidad. El comportamiento del consumidor indica que la textura es uno de los factores más influyentes para determinar la calidad de esta, el cual es dependiente de elementos tales como la ternura y el grado de maduración. Técnicamente el US puede actuar de dos maneras en el tejido de la carne: por romper la integridad de las células musculares y promoviendo reacciones enzimáticas (Alarcon-Rojo et al., 2015), mientras que algunos autores (Jayasooriya et al., 2004) afirman que la exposición prolongada a las ondas ultrasónicas de alta intensidad provoca un ablandamiento significativo de la carne.

Por otra parte Jayasooriya et al., (2007), estudiaron el efecto de US en 2 especies de bovino, reportando que la dureza estuvo afectada por el tratamiento con US ( $p<0,0001)$ a los 50, 100, 150, 200 y 250 seg de exposición, siendo el control $21 \%$ y $16 \%$ mayor que la muestra tratada a US a los 50 seg y 250 seg respectivamente. La textura es una propiedad importante que determina la calidad sensorial de los alimentos, Zhao et al., (2014) estudiaron el efecto del US sobre las propiedades funcionales de gel compuesto de proteína miofibrilar de pechuga de pollo en el cual todas las muestras tratadas con US fueron más altos que los del control $(p<0,05)$, especialmente la dureza y la masticabilidad que aumentó de $93,3 \%$ a $120,33 \%$ y $110,75 \%$ al $140,09 \%$ respectivamente. Como resultado del rápido movimiento molecular causado por la cavitación y el desarrollo del radical hidroxilo de la hidrolisis del agua que aumenta las reacciones químicas y modifica otras moléculas. También McDonell et al., (2014), aplicaron el uso de US de potencia para acelerar el curado de carne, indicando que no hubo diferencias $(p>0,05)$ en la dureza, masticabilidad y elasticidad de las muestras, con valores medios de $166 \pm 13,17 \mathrm{~N} ; 364 \pm 39,89$ y $4,96 \pm 0,2$ respectivamente. La cohesión de las muestras se redujo $(p \leq 0,05)$ para todos los tratamientos ultrasónicos en comparación con el control con diferencias cercanas al 5,6\% (McDonell et al., 2014).

\section{Medición de color en la carne expuesta a US}

En la Tabla 2 se evidencia el análisis de varianza ANOVA, al igual que la prueba DMS para la comparación de medias para cada uno de los diferentes tiempos de exposición aplicados. En los parámetros del color se muestran los promedios, y las barras de error \pm representan la desviación estándar. Se observa que hubo diferencias significativas en los parámetros de luminosidad (blanco / negro), indicando que el US tuvo un efecto significativo en la muestra tratada a diferentes tiempos de exposición $(p<0,05)$. En relación a luminosidad $L^{*}$, se presenta una reducción variable en las muestras tratadas durante 3,4 y 5 min, lo cual indica que estas se tornan más oscuras. En el análisis de los datos se encontró que los valores más bajos 
de $L^{*}$ corresponde al tratamiento durante $5 \mathrm{~min}$, el cual representa un color más oscuro, y los valores más alto de $L^{*}$ fue el control que significa un color claro. Un comportamiento similar reportaron Dolatowski y Stadnik (2008) en los parámetros de $L^{*}$, el cual mostraron diferencias significativas a las $24 h(p<0,05)$, aumentando el $3 \%$ respecto al control en la carne de vacuno expuesta a US. También se observa que el índice $a^{*}$ no tuvo diferencias significativas entre la muestra control y el tratamiento durante 3min, expresando que el US no afectó la muestra, sin embargo a los $4 \mathrm{~min}$ y $5 \mathrm{~min}$ hubo un efecto significativo $(p<0,05)$ entre los tratamientos, indicando que el color rojo se hizo menos intenso. Los únicos parámetros más afectados fueron las coordenadas colorimétricas $a^{*} y b^{*}$, el cual tuvieron un efecto significativo $(p<0,05)$ en todas las muestras sometidas a US con respecto al control, con valores menores del $18,63 \%$ y $18,90 \%$ respectivamente, esto indica que el US afecta estos índices del color en las muestras tratadas con esta tecnología.

Tabla 2: Efecto del US en el color de la carne. Letras diferentes expresan diferencias estadísticamente significativas, $p<0,05$

\begin{tabular}{|c|c|c|c|c|}
\hline \multicolumn{2}{|c|}{ Atún } & \multicolumn{3}{|c|}{ Parámetros del color } \\
\hline \multirow{5}{*}{ 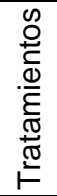 } & Parámetros & $\mathrm{L}^{*}$ & $a^{*}$ & $b^{\star}$ \\
\hline & Control US & $48,3 \pm 0,2^{a}$ & $9,46 \pm 0,0^{a}$ & $16,4 \pm 0,0^{a}$ \\
\hline & US 3 minutos & $44,9 \pm 1,1^{\mathrm{C}}$ & $8,89 \pm 0,5^{\mathrm{a}}$ & $15,1 \pm 0,6^{b}$ \\
\hline & US 4 minutos & $47,8 \pm 0,1^{b}$ & $5,90 \pm 0,3^{c}$ & $13,7 \pm 0,2^{c}$ \\
\hline & US 5 minutos & $43,1 \pm 0,0^{\mathrm{d}}$ & $8,30 \pm 0,7^{0}$ & $11,1 \pm 1,5^{\mathrm{d}}$ \\
\hline
\end{tabular}

Investigadores como Stadnik et al., (2011) estudiaron el efecto de US en carne de vacuno durante 120s, reportando que el parámetro $L^{*}$ en el paso del tiempo no tuvo influencia $(p>0,05)$, mientras que los valores de $a^{*}$ y $b^{*}$ únicamente hubo diferencias significativas a las $48 \mathrm{~h}$ y $24 \mathrm{~h}$ respectivamente. De acuerdo con la literatura, el US es capaz de producir modificaciones en los parámetros de textura, color y nutrientes (Pringret et al., 2013). En términos generales, estos parámetros aumentaron, lo que indica que el proceso de secado por US activado causó la coloración amarillenta (mayor $b^{*}$ ) de las muestras y el aumento de su índice de luminosidad ( $L^{*}$ superior) (Ozuna et al., 2014). Por su parte Campo y Gélvez (2011) estudiaron el efecto de la termosonicación sobre las propiedades fisicoquímicas del hongo comestible (Pleurotus ostreatus), en donde el cambio visual de color guardó relación con los parámetros determinados a partir de los valores de $L^{*}$, $a^{*}$ y $b^{*}$ en el cual durante el almacenamiento las muestras tratadas con US se redujo el parámetro de luminosidad $\left(\mathrm{L}^{*}\right)$, indicando que la muestra se oscureció y el cambio de color se incrementó.

\section{Efecto de los CM sobre el pH de la carne de lomo de atún.}

En la Figura 2 se observa el efecto de la aplicación de CM sobre la variación del pH en la carne de lomo de atún (Thunnus albacares). Las letras a, b, c y d indican los tiempos de exposición a CM. Como se observa, este tratamiento produce un aumento significativo $(p<0,05)$ del $\mathrm{pH}$, el cual no es lineal con respecto al tiempo de exposición de los tratamientos.

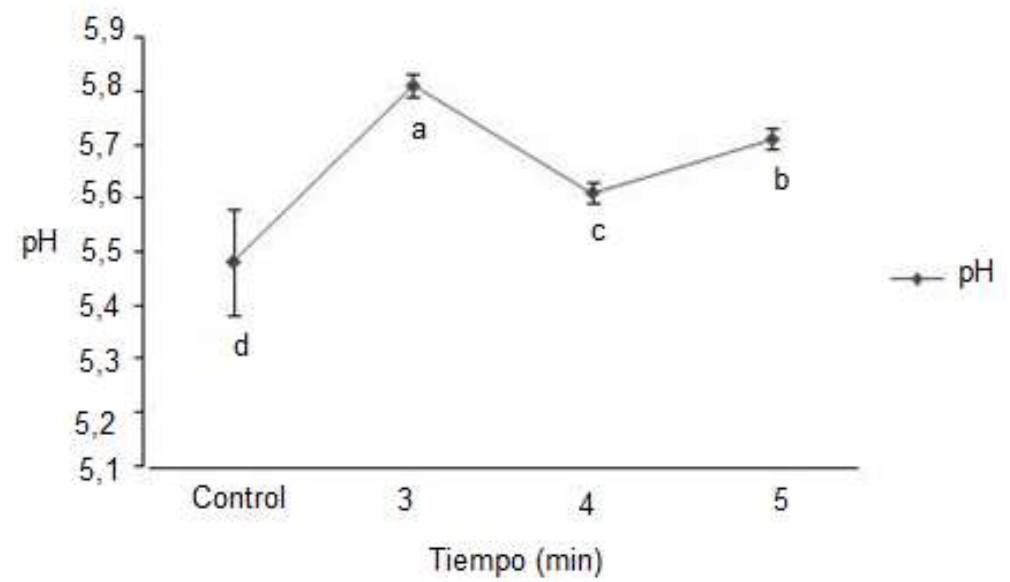

Fig. 2: Efecto del campo magnético sobre el pH de la carne de lomo de atún

En el análisis estadístico realizado, se presentan diferencias significativas entre todas las muestras de carne de atún. Conforme a la prueba de DMS, se establecieron 4 grupos homogéneos. El primero representado por el control y los otros tres constituidos por las muestras tratadas con CM. El aumento del pH por el tratamiento de CM se ve afectado con énfasis en su actividad alcalina, influenciado por las condiciones del $\mathrm{pH}$ inicial (Mulay, 1964). A diferencia de lo que ocurre con el US, en este caso los valores de pH siempre se encuentran por encima del control, hecho que puede estar relacionado con el tipo de fuerza que se aplica a 
la muestra, teniendo en cuenta que el $\mathrm{pH}$ de una sustancia se debe a la concentración de iones hidronio que son de carga positiva y de poca masa atómica, es posible que se congregaran de tal manera que al momento de medir el pH, los iones hidronio no estuvieran en disolución, por tanto no ejercían valores más representativos (Mulay, 1964). En estudios realizados por Romero y Gélvez (2013) en una emulsión de carne de Búfalo (Bubalus bubalis), mostraron que el $\mathrm{pH}$ no tuvo diferencias significativas $(\mathrm{p}<0,05)$ a los 3 y 5 min de exposición a CM con valores de $5,77 \pm 0,0020^{b}$ y $5,78 \pm 0,010^{b}$ respectivamente, sin embargo el tratamiento durante $10 \mathrm{~min}$ tuvo diferencias significativas $(p>0,05)$ respecto al control y a los otros tiempos de intensidad.

\section{Efecto de los CM sobre la textura de la carne de lomo de atún}

El efecto del tratamiento en el perfil de textura en la carne de lomo de atún se observa en la Tabla 3 . En esta se detalla los resultados del análisis de varianza y la comparación de medias a través de la prueba DMS para las variables analizadas. Los subíndices a, b, c y d muestran las diferencias significativas en los parámetros de textura. En las características de dureza y elasticidad se observa que la carne expuesta a $\mathrm{CM}$ tuvo un efecto significativo con relación al control $(\mathrm{p}<0,05)$, mostrando que los $\mathrm{CM}$ afectó el parámetro de dureza siendo menor que el control en un 37,7\%. Por su parte la característica de elasticidad del control en promedio fue menor que los otros tratamientos en un $65,46 \%$. También se detalla que la dureza y la cohesividad no tuvieron diferencias significativas entre los tratamientos realizados a diferentes tiempos $(p>0,05)$. Por otra parte la masticabilidad en la muestra control y los CM durante 4 min no tuvo un efecto significativo, no obstante a los $3 \mathrm{~min}$ y $5 \mathrm{~min}$ las muestras fueron estadísticamente significativas con respecto a los otros dos tratamientos $(p>0,05)$.

Tabla 3: Efecto de los CM en la textura de la carne de lomo de atún. DMS=Diferencia mínima significativa. Diferentes letras expresan diferencias significativas a $p<0,05$

\begin{tabular}{|c|c|c|c|c|}
\hline \multirow{2}{*}{ Tratamiento } & \multicolumn{4}{|c|}{ Parámetros del análisis de perfil de textura } \\
\cline { 2 - 5 } & Dureza $(\mathrm{N})$ & Cohesividad & Elasticidad & Masticabilidad $(\mathrm{Kg})$ \\
\hline Control & $73,04 \pm 5,1^{\mathrm{a}}$ & $0,18 \pm 0,00^{\mathrm{a}}$ & $1,05 \pm 0,13^{\mathrm{a}}$ & $0,23 \pm 0,00^{\mathrm{a}}$ \\
\hline CM 3 min & $43,12 \pm 8,6^{\mathrm{b}}$ & $0,13 \pm 0,33^{\mathrm{a}}$ & $3,19 \pm 0.55^{\mathrm{b}}$ & $0,13 \pm 0,00^{\mathrm{b}}$ \\
\hline CM 4 min & $48,06 \pm 6,9^{\mathrm{b}}$ & $0,11 \pm 0,28^{\mathrm{a}}$ & $2,97 \pm 0,62^{\mathrm{b}}$ & $0,23 \pm 0,00^{\mathrm{a}}$ \\
\hline CM 5 min & $45,31 \pm 5,3^{\mathrm{b}}$ & $0,10 \pm 0,31^{\mathrm{a}}$ & $2,97 \pm 0,71^{\mathrm{b}}$ & $0,16 \pm 0,00^{\mathrm{c}}$ \\
\hline Valor-p & 0,0010 & 0,9825 & 0,0027 & 0,0000 \\
\hline DMS, $\alpha=0,05$ & 61,91 & 0,54 & 3,19 & 0,19 \\
\hline
\end{tabular}

Estudios realizados por Romero y Gélvez (2013) en una emulsión de carne de Búfalo con tiempos de exposición a CM de 0,3, 5 y 10min, se observó que la dureza no tuvo diferencias significativas entre la muestra control y el tratamiento durante $3 \mathrm{~min}$, pero si se evidenció un efecto a los $5 \mathrm{~min}$ y $10 \mathrm{~min}$ de exposición a CM $(\mathrm{p}<0,05)$. Por otra parte la elasticidad no tuvo diferencias significativas en la muestra control y CM durante $5 \mathrm{~min}$ y $10 \mathrm{~min}$, sin embargo d urante los primeros $3 \mathrm{~min}$ se observaron diferencias estadísticamente significativas $(p<0,05)$, siendo en promedio mayor que las demás muestras analizadas en un $9,76 \%$. También se observó que la masticabilidad no tuvo diferencias significativas entre la muestra control y los dos primeros tiempos de exposición a CM $(p<0,05)$, sin embargo a los 10min este parámetro aumento en promedio en un 33,3\% respecto a las demás muestras. Los resultados sobre el efecto de los $\mathrm{CM}$ de alta intensidad sobre los microorganismos son escasos e inconsistentes, mientras que algunos reportan efectos de inactivación (Hoffman, 1985), otros reportan ningún efecto (Malko et al., 1994) o las tasas de crecimiento microbiano (Van Nostran et al., 1967). Investigaciones exploratorias no encontraron ningún efecto de E. coli y S. cerevisiae con CM estáticos (San Martín et al., 2001b). Por otra parte Strašák et al., (2002) estudiaron el efecto de CM de baja frecuencia en la bacteria Escherichia coli, reportando que el número de unidades formadoras de colonia disminuyeron de manera exponencial con el tiempo de exposición de $1 \mathrm{~h}$.

\section{Medición de color de la carne sometida a CM}

En la Tabla 4 se observa el ANOVA realizado en los parámetros de color y la prueba DMS para comparaciones de medias. Se observa que todas las muestras tratadas con CM tuvieron diferencias significativas $(p<0,05)$ en los valores de $L^{*}, a^{*}$ y $b^{*}$, sin embargo a los 5 min de tratamiento no se observaron diferencias respecto al control. En las coordenadas colorimétricas del color $L^{*}, a^{*}, y$ b* presentó una disminución durante los 3 y 4 minutos en las muestras tratadas con CM respecto a la muestra control, observándose diferencias significativas con los demás tratamientos $(p<0,05)$. Respecto al índice $L^{*}$, $a^{*}$ y $b^{*}$ las muestras tratadas con CM en promedio fueron menor que el control en un 6,14\%, 19,87\% y $17,03 \%$ respectivamente, observándose que el parámetro $L^{*}$ fue el que se vio menos afectado. Es decir la aplicación de este tipo de tecnología es más adecuada cuándo los tiempos de exposición superan lo $4 \mathrm{~min}$ de tratamiento. 
Tabla 4: Efecto del campo magnético en el color de la carne. Diferentes letras expresan diferencias significativas a $p<0,05$

\begin{tabular}{|c|c|c|c|c|}
\hline \multicolumn{2}{|c|}{ Atún } & \multicolumn{3}{|c|}{ Parámetros del color } \\
\hline \multirow{5}{*}{ 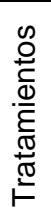 } & Parámetros & $L^{*}$ & $a^{*}$ & $b^{*}$ \\
\hline & Control CM & $48,3 \pm 0,2^{a}$ & $9,46 \pm 0,0^{a}$ & $16,4 \pm 0,0^{a}$ \\
\hline & CM 3 minutos & $44,6 \pm 0,4^{b}$ & $8,03 \pm 0,2^{b}$ & $13,3 \pm 0,4^{b}$ \\
\hline & CM 4 minutos & $44,2 \pm 0,3^{\mathrm{c}}$ & $6,00 \pm 0,9^{c}$ & $11,3 \pm 0,5^{\mathrm{C}}$ \\
\hline & CM 5 minutos & $47,2 \pm 0,8^{a}$ & $8,71 \pm 0,2^{a}$ & $16,2 \pm 0,7^{a}$ \\
\hline
\end{tabular}

Romero y Gélvez (2013) reportaron diferencias significativas entre la muestra control y los demás tratamientos en carne de búfalo $(p<0,05)$, sin embargo los diferentes tiempos de exposición a CM no fueron estadísticamente significativo $(p>0,05)$. Para el índice $a^{*}$ que representa la escala de color rojo, y el índice amarillo $b^{*}$, a mayor tiempo de exposición disminuía los valores de estos parámetros, no habiendo diferencias significativas en los dos primeros tiempos de CM, pero si en las demás muestras analizadas.

\section{CONCLUSIONES}

La aplicación de ultrasonido en la carne de atún afectó significativamente el $\mathrm{pH}$, en cambio la muestra tratada con campos magnéticos mostró un comportamiento en forma no lineal al transcurrir el tiempo. El perfil de textura del lomo de atún se afectó con la aplicación de ambos tratamientos, de forma similar las características texturales de las muestras tratadas como es: la dureza cohesividad y masticabilidad, disminuyeron con los diferentes tiempos de exposición, mientras que el parámetro de la elasticidad aumentó. En las coordenadas colorimétricas del color, las muestras tratadas con las dos tecnologías por separado, se vieron afectadas, disminuyendo para todos los casos.

\section{REFERENCIAS}

Alarcon-Rojo, A.D. y otros cuatro autores, Power ultrasound in meat processing, Meat science, 107, 86-93 (2015)

A.O.A.C., Officials Methods of Analysis (971.09), 12a Ed, Association of Official Analytical Chemists, U.S.A, pp 1094 (1990)

Arroyo, C. y otros tres autores, Inactivation of Cronobacter sakazakii by ultrasonic waves under pressure in buffer and foods, International Journal of Food Microbiology, 144(3), 446-454 (2011)

Barbosa-Cánovas, G.V. y otros cinco autores, Introduction to Innovative Food Processing Technologies: Background, Advantages, Issues and Need for Multiphysics Modeling, Innovative Food Processing Technologies: Advances in Multiphysics Simulation, 3-21 (2011)

Barbosa-Cánovas, G. y D. Bermúdez-Aguirre, Procesamiento no térmico de alimentos, Scientia Agropecuaria, 1(1), 81-93 (2010)

Baque-Menoscal, J., D., Páez-Rosas y M. Wolff, Hábitos alimentarios de dos peces pelágicos Thunnus albacares y Acanthocybium solandri de la Reserva Marina de Galápagos. Revista de biología marina y oceanografía, 47(1), 01-11 (2012)

Bilek, S.E., y F. Turantaş, Decontamination efficiency of high power ultrasound in the fruit and vegetable industry, a review, International journal of food microbiology, 166(1), 155-162 (2013)

Boufleur, L.A., y otros cinco autores, Elemental characterization of Brazilian canned tuna fish using particle induced X-ray emission (PIXE), Journal of Food Composition and Analysis, 30(1), 19-25 (2013)

Campo, Y. y V.M. Gélvez, Efecto de la termosonicaciòn sobre las propiedades fisicoquímicas del hongo comestible (Pleurotus ostreatus) fresco empacado al vacio, Bistua:Revista de la Facultad de Ciencias Básicas, 9(2), 55-63 (2011)

Condón, S., P. Mañas y G. Cebrián, Manothermosonication for microbial inactivation, In Ultrasound technologies for food and bioprocessing, Springer, New York. pp. 287-319 (2011) 
Chen, L. y U.L. Opara, Texture measurement approaches in fresh and processed foods-A review, Food Research International, 51(2), 823-835 (2013)

Chen, J. y otros cinco autores, Intervention technologies for ensuring microbiological safety of meat: current and future trends, Comprehensive Reviews in Food Science and Food Safety, 11(2), 119-132 (2012)

Evelyn, F. y V.M. Silva, Use of power ultrasound to enhance the thermal inactivation of Clostridium perfringens spores in beef slurry, International journal of food microbiology, 206, 17-23 (2015)

FAO, Food and Agriculture Organization, Agricultura mundial: hacia los años (en línea, http://www.fao.org/docrep/004/y3557s/y3557s00.htm. Acceso: 20 de febrero, 2015)

FDA., Food and Drug Administration, Kinetics of Microbial Inactivation for Alternative Food Processing

Technologies -- Oscillating Magnetic Fields, (en línea:

http://www.fda.gov/Food/FoodScienceResearch/SafePracticesforFoodProcesses/ucm103131.htm, Acceso

18 Julio 2015. (2000)

Haughton, P. N. y otros seis autores, An evaluation of the potential of high-intensity ultrasound for improving the microbial safety of poultry, Food and bioprocess technology, 5(3), 992-998 (2012)

Hernández, S. y V.M. Gélvez, Uso del ultrasonido y su influencia en las propiedades del huevo entero líquido, @limentech ciencia y tecnología alimentaria, 9(2), 132-144 (2011)

Hofmann, G.A., Deactivation of microorganisms by an oscillating magnetic field. Patent NumberUS Patent 4, 524, 079 (1985)

Jayasooriya, S.D. y otros tres autores, Effect of high power ultrasound waves on properties of meat: a review, International Journal of Food Properties, 7(2), 301-319 (2004)

Jayasooriya, S.D. y otros tres autores, Effect of high power ultrasound and ageing on the physical properties of bovine Semitendinosus and Longissimus muscles, Meat Science, 75(4), 628-639 (2007)

Jump, D.B., C.M. Depner y S. Tripathy, Omega-3 fatty acid supplementation and cardiovascular disease Thematic Review Series: New lipid and lipoprotein targets for the treatment of cardiometabolic diseases, Journal of lipid research, 53(12), 2525-2545 (2012)

Malko, J.A. y otros tres autores, Search for influence of 1.5 Tesla magnetic field on growth of yeast cells, Bioelectromagnetics, 15(6), 495-501 (1994)

McDonnell, C.K. y otros tres autores, The effect of ultrasonic salting on protein and water-protein interactions in meat, Food chemistry, 147, 245-251 (2014)

Mesa; Y. y otros tres autores, Efecto de un campo electromagnético de 40 Gaus a $60 \mathrm{~Hz}$ en la viabilidad del cultivo bioyogurt en las fases de crecimiento bacteriana, Investigación y Saberes, 1(3), 65-70 (2012)

Montgomery, D.C. Diseño y análisis de experimentos, $2^{a}$ edición, 60-65, Limusa Wiley. México, México (2004)

Mulay, L.N., Basic concepts related to magnetic fields and magnetic susceptibility biological effects of magnetic fields (M. F. Barnothy, ed.), Plenum Press, New York, Vol. 1, pp. 33-55 (1964)

Ozuna, C. y otros tres autores, Low-temperature drying of salted cod (Gadus morhua) assisted by high power ultrasound: Kinetics and physical properties, Innovative Food Science and Emerging Technologies, 23, 146-155 (2014)

Patist, A. y D. Bates, Industrial applications of high power ultrasonics. In Ultrasound Technologies for Food and Bioprocessing, Springer, New York, pp. 599-616 (2011)

Pereira, R.N. y A.A Vicente, Environmental impact of novel thermal and non-thermal technologies in food processing, Food Research International, 43(7), 1936-1943 (2010)

Pingret, D., A.S. Fabiano-Tixier y F. Chemat, Degradation during application of ultrasound in food processing, a review, Food Control, 31(2), 593-606 (2013) 
Rastogi, N.K., Opportunities and challenges in nonthermal processing of foods, Innovation in food engineering, New techniques and products: 3-58 (2010)

Romero, P.E. y V.M. Gélvez, Efecto de los campos magnéticos y el ultrasonido sobre la calidad microbiológica y las propiedades funcionales en una emulsión de carne de bufalo (Bubalus bubalis), Bistua: Revista de la Facultad de Ciencias Básicas, 11(1), 67-76 (2013)

San Martín, M.F. y otros cuatro autores, Inactivation effect of an 18-T pulsed magnetic field combined with other technologies on Escherichia coli, Innovative Food Science \& Emerging Technologies, 2(4), 273-277 (2001a)

San Martin, M.F., y otros cuatro autores, Inactivation effect of $18 \mathrm{~T}$ static and pulsed magnetic fields on Escherichia coli and Saccharomyces cerevisiae, Innovative Food Sci. Emerg. Technol, 2, 273-277 (2001b)

Stadnik, J. y Z.J. Dolatowski, Influence of sonication on Warner-Bratzler shear force, colour and myoglobin of beef (m. semimembranosus), European Food Research and Technology, 233(4), 553-559 (2011)

Stadnik, J. y Z.J. Dolatowski, Influence of sonification on the oxidative stability of beef, In 54 th International Congress of Meat Science and Technology, 10, 1-3 (2008)

Strašák, L., V., Vetterl. y J. Šmarda., Effects of low-frequency magnetic fields on bacteria Escherichia coli, Bioelectrochemistry, 55(1), 161-164 (2002)

Turantaş, F., G.B. Kılıç y B. Kılıç, Ultrasound in the meat industry: General applications and decontamination efficiency, International Journal of Food Microbiology, 198, 59-69 (2015)

Van Nostran, F.E., R.J., Reynolds, y H.G. Hedrick, Effects of a high magnetic field at different osmotic pressures and temperatures on multiplication of Saccharomyces cerevisiae, Applied microbiology, 15(3), 561-563 (1967)

Villalpanda, M. A., T.M., Armenteros y C.M. Fiallo, El campo magnético aplicado a la industria alimentaria. Publitec .s.a Argentina, 1-8 (2011)

Zhao, Y.Y. y otros seis autores, Effect of pre-emulsification of plant lipid treated by pulsed ultrasound on the functional properties of chicken breast myofibrillar protein composite gel, Food Research International, 58(1), 98-104 (2014) 